\title{
Asymmetric Information on Price Can Affect Bertrand Duopoly Players with the Gradient-Based Mechanism
}

\author{
S. S. Askar iD ${ }^{1,2}$ \\ ${ }^{1}$ Department of Statistics and Operations Research, College of Science, King Saud University, Riyadh 11451, Saudi Arabia \\ ${ }^{2}$ Department of Mathematics, Faculty of Science, Mansoura University, Mansoura 35516, Egypt \\ Correspondence should be addressed to S. S. Askar; s.e.a.askar@hotmail.co.uk
}

Received 26 October 2020; Revised 22 November 2020; Accepted 24 November 2020; Published 10 December 2020

Academic Editor: Adel Ouannas

Copyright (c) 2020 S. S. Askar. This is an open access article distributed under the Creative Commons Attribution License, which permits unrestricted use, distribution, and reproduction in any medium, provided the original work is properly cited.

\begin{abstract}
We study a Bertrand duopoly game in which firms adopt a gradient-based mechanism to update their prices. In this competition, one of the firms knows somehow the price adopted by the other firm next time step. Such asymmetric information of the market price possessed by one firm gives interesting results about its stability in the market. Under such information, we use the bounded rationality mechanism to build the model describing the game at hand. We calculate the equilibrium points of the game and study their stabilities. Using different sets of parameter values, we show that the interior equilibrium point can be destabilized through flip and Neimark-Sacker bifurcations. We compare the region of stability of the proposed model with a classical Bertrand model without asymmetric information. The results show that the proposed game's map is noninvertible with type $Z_{0}-Z_{2}$ or $Z_{1}-Z_{3}$, while the classical model is of type $Z_{0}-Z_{2}$ only. This explains the quite complicated basins of attraction given for the proposed map.
\end{abstract}

\section{Introduction}

The Bertrand competition was introduced as a model describing an economic game by the famous scientist Joseph Louis François Bertrand [1]. Such game was used in the literature to simulate interactions among firms (players) who set prices as their strategies. Bertrand claimed but not formalized that firms set prices than quantities as their outputs in the competition would take place with prices and marginal costs that are equal. Comparing Cournot games (on where players set quantities as their strategies) with Bertrand, we report few studies on Bertrand games in the literature. For instance, the minimal differentiation principle was tested in a Bertrand competition in [2], and it was found that it was applied to spatial competition. The capacity precommitment as an entry-deterring device was reexamined in [3] on the price competition of Bertrand-Edgeworth model. In [4], a repeated game of price was studied to detect that when the number of competing firms increased above two competitive players, the outcomes become less likely. For the case of spatial discrimination, a comparison between Bertrand and Cournot in the context of spatial duopoly was analyzed in [5]. In [6], a Bertrand game with an uncertain number of active firms was analyzed. In the case of differentiated quantities, a price setting game has been analyzed in a continuous time scale with random demands [7].

All the above reported studies handled the price setting game statically. There are few studies of Bertrand games about the complex dynamic characteristics of their equilibrium points. We report some of them from the literature as follows. In [8], a Bertrand game whose players adopted the bounded rationality mechanism to update their prices investigated that bifurcation and chaos may occur when the speed of adjustment of players also increases. $\mathrm{Ma}$ and $\mathrm{Wu}$ [9] introduced the game of Bertrand triopoly with bounded rational players and investigated that the time delay may not improve the stability region of the game. $\mathrm{Ma}$ and Sun studied the multiteam Bertrand game in [10]. In [11], a Bertrand game with delayed bounded rational players was considered. In that study, it was pointed out that lagged structure may affect the stable region of the stable state. For substitutable 
products in a supply chain [12], the game of price setting analyzed the influences of different competitive strategies on optimum decisions of prices. In [13], an advertising cost-dependent demand for a two-echelon supply chain was investigated, and the obtained results showed that it was beneficial for the firms to use different wholesale pricing strategies. With differentiated products, the complex dynamic characteristics of a Bertrand game were discussed by Fanti et al. in [14]. They have deduced that the interior fixed point can be destabilized when differentiation between firms increased, and therefore, chaotic attractors with complicated structure may arise. Other studies on Bertrand and Cournot-Bertrand games and more information on their complex dynamic behaviors have been reported in the literature $[15,16]$. We should also highlight some recent studies on the Bertrand game. For instance, in [17], the bounded rationality and naive expectation mechanisms were used to study a dynamic model of the quantum Bertrand game with differentiated products. In [18], a Bertrand game in the downstream market was investigated. A triopoly Bertrand game based on differentiated products was investigated in [19]. A Cournot-Bertrand game was introduced and studied in [20]. In [21], necessary and sufficient conditions for a unique Nash equilibrium in a standard Bertrand duopoly game based on homogeneous products were analyzed.

There are different types of adjustment mechanisms that have been adopted to model the maps describing such games. For instance, there are the gradient-based mechanisms such as bounded rationality. Bounded rationality has been intensively used for this purpose in the literature [22-27]. This mechanism depends on the estimation of players' profits for updating their outputs. If the profits are increased (or decreased), this will affect the prices whether they will be increased (or decreased). There are also other mechanisms that have been used to formulate the Bertrand and Cournot games such as the local monopolistic approximation (LMA) mechanism [28] and tit-for-tat approach [29]. In the paper at hand, we recall the bounded rationality mechanism to formulate our Bertrand game.

The current paper belongs to the research direction of the Bertrand game with players adopting the bounded rationality, and one of the players possesses information about the price its opponent adopts. We model the game by introducing a discrete-time dynamical map whose variables are prices, and it is nonlinear. The main results in this paper focus on many things. We concern with the destabilization of the interior equilibrium and the routes responsible for that. We investigate the flip and Neimark-Sacker bifurcations under different types of parameter sets causing chaotic behavior of the game's map. Depending on a rich numerical analysis, we show different types of quite complicated attractive basins of periodic cycles. Furthermore, the noninvertibility aspect is numerically discussed for the map.

The paper presents the map describing the game in Section 2. In Section 3, we discuss the main results which include investigation of the stability of the equilibrium points of the map. It also gives local and global analyses about the interior equilibrium point and the routes by which it can be destabilized. This section includes numerical simulation about the basins of attraction and their complicated structure. We also prove in the same section that the game's map is a noninvertible map of type $Z_{0}-Z_{2}$ or $Z_{1}-$ $Z_{3}$ depending on the nature of preimages coexisted. Furthermore, in this section, we compare the influences of asymmetric price information with the classical model without such information. Finally, the obtained results are concluded in Section 4.

\section{The Model}

The model in this paper consists of two firms (players) whose products are differentiated, and their prices are derived from the utility function introduced by Singh and Vives [30] as follows:

$$
U\left(q_{1}, q_{2}\right)=a\left(q_{1}+q_{2}\right)-\frac{1}{2}\left(q_{1}^{2}+q_{2}^{2}+2 b q_{1} q_{2}\right), \quad q_{1}, q_{2}>0,
$$

where $a$ is a constant and $b$ is restricted in the interval $(-1,1)$. Both $q_{1}$ and $q_{2}$ are the quantities produced by each firm. The following characterize this utility:

(1) $U$ is concave which means $\left(\partial^{2} U / \partial q_{i}^{2}\right)=-1<0 ; i=1,2$. Economically, the marginal utility of each quantity is decreasing.

(2) $\left(\partial^{2} U / \partial q_{1} q_{2}\right)=-b \neq 0$. This means that the marginal utility of quantity depends on the other.

(3) $U\left(\theta q_{1}, \theta q_{2}\right) \neq \theta U\left(q_{1}, q_{2}\right)$. This means it is not homogeneous.

(4) Using a budget constraint $\sum_{i=1}^{2} p_{i} q_{i} \leq m$, we get

$$
\begin{aligned}
& \operatorname{Max} U\left(q_{1}, q_{2}\right), \\
& \text { s.t. } \quad \sum_{i=1}^{2} p_{i} q_{i} \leq m .
\end{aligned}
$$

Problem (2) is a maximization problem with a consumer's budget constraint. $p_{i}$ denotes the price of quantity $q_{i}$, and $m$ is a constant. Solving (2) gives the following demand functions:

$$
\begin{aligned}
& q_{1}=\frac{a}{1+b}-\frac{p_{1}}{1-b^{2}}+\frac{b p_{2}}{1-b^{2}} \\
& q_{2}=\frac{a}{1+b}-+\frac{b p_{1}}{1-b^{2}}-\frac{p_{2}}{1-b^{2}} .
\end{aligned}
$$

We should mention here that $b$ denotes the horizontal differentiation degree. If $b=0$, we have two monopolistic firms, and $b=1$ represents two identical firms. Negative values for $b$ refer to complementarity between the firms. Assuming linear costs, $C_{i}\left(q_{i}\right)=c_{1} q_{i}$, where $\left(\partial C_{i} / \partial q_{i}\right)=c_{i}$ is a constant marginal cost. Now, the profit of each firm becomes 


$$
\begin{aligned}
& \pi_{1}=\left(p_{1}-c_{1}\right) q_{1}=\left(p_{1}-c_{1}\right)\left(\frac{a}{1+b}-\frac{p_{1}}{1-b^{2}}+\frac{b p_{2}}{1-b^{2}}\right) \\
& \pi_{2}=\left(p_{2}-c_{2}\right) q_{1}=\left(p_{1}-c_{1}\right)\left(\frac{a}{1+b}-+\frac{b p_{1}}{1-b^{2}}-\frac{p_{2}}{1-b^{2}}\right) .
\end{aligned}
$$

Now, we consider a Bertrand game whose players adopt a gradient-based mechanism in order to update their prices according to the following discrete dynamical map:

$$
\begin{aligned}
& p_{1, t+1}=p_{1, t}+k p_{1, t} \frac{\partial \pi_{1}\left(p_{1, t}, p_{2, t}\right)}{\partial p_{1}} \\
& p_{2, t+1}=p_{2, t}+k p_{2, t} \frac{\partial \pi_{2}\left(p_{1, t+1}, p_{2, t}\right)}{\partial p_{2}} .
\end{aligned}
$$

It should be noted that the second equation in (5) differs from the first because the second player in the game has some advantages. He knows by some way the price of the first player next time step. This kind of asymmetric information gives with (4) and (5) the following discrete time map:

$$
T\left(p_{1}, p_{2}\right):\left\{\begin{array}{l}
p_{1, t+1}=p_{1, t}+\frac{k p_{1, t}}{1-b^{2}}\left[a(1-b)+c_{1}-2 p_{1, t}+b p_{2, t}\right] \\
p_{2, t+1}=p_{2, t}+\frac{k p_{2, t}}{1-b^{2}}\left[a(1-b)+c_{2}+b p_{1, t}-2 p_{2, t}\right] \\
+\frac{k^{2} b p_{1, t} p_{2, t}}{\left(1-b^{2}\right)^{2}}\left[a(1-b)+c_{1}-b c_{2}-2 p_{1, t}+2 b p_{2, t}\right] .
\end{array}\right.
$$

Map (6) is nonlinear, and due to the asymmetric information possessed by the second firm, it becomes more complex. Without this asymmetric information, we get the following map:

$$
T_{1}\left(p_{1}, p_{2}\right):\left\{\begin{array}{l}
p_{1, t+1}=p_{1, t}+\frac{k p_{1, t}}{1-b^{2}}\left[a(1-b)+c_{1}-2 p_{1, t}+b p_{2, t}\right] \\
p_{2, t+1}=p_{2, t}+\frac{k p_{2, t}}{1-b^{2}}\left[a(1-b)+c_{2}+b p_{1, t}-2 p_{2, t}\right] .
\end{array}\right.
$$

In this paper, we will compare between maps (6) and (7) in order to see the influences of asymmetric information on the complex dynamic characteristics of the game's equilibrium points.

\section{Main Results of Map (6)}

3.1. Fixed Points and Stability. Map (6) has four fixed points:

$$
\begin{aligned}
& E_{0}=(0,0), \\
& E_{1}=\left(0, \frac{a(1-b)+c_{2}}{2}\right), \\
& E_{2}=\left(\frac{a(1-b)+c_{1}}{2}, 0\right), \\
& E_{3}=\left(\widehat{p}_{1}, \widehat{p}_{2}\right),
\end{aligned}
$$

where

$$
\begin{aligned}
\widehat{p}_{1} & =\frac{1}{4 k b^{2}}\left[A-B_{1} k+\sqrt{B_{1}^{2} k^{2}+D k+A^{2}}\right], \\
\widehat{p}_{2} & =\frac{1}{4 k b^{2}}\left[A+B_{2} k+\sqrt{B_{1}^{2} k^{2}+D k+A^{2}}\right], \\
A & =\left(1-b^{2}\right)\left(4-b^{2}\right), \\
B_{1} & =b^{2}\left[\left(a-c_{2}\right) b-a-c_{1}\right], \\
B_{2} & =b^{2}\left[\left(a+c_{2}\right) b-a-c_{1}\right], \\
D & =2 b^{2}\left(1-b^{2}\right)\left[a b^{3}-c_{2} b^{3}+3 a b^{2}-c_{1} b^{2}-4 a-4 c_{1}\right] .
\end{aligned}
$$

The points $E_{0}, E_{1}$, and $E_{2}$ are called corner fixed points, while $E_{3}$ is an interior fixed point. It is clear that $E_{0}, E_{1}$, and $E_{2}$ are positive since $-1<b<1$. Due to the complicated form of the interior point, we will discuss its positivity and stability by assuming values for the parameters $a, b, c_{1}, c_{2}$, and $k$. The Jacobian of map (6) becomes

$$
J_{m}=\left(\begin{array}{ll}
J_{11} & J_{12} \\
J_{21} & J_{22}
\end{array}\right),
$$

where

$$
\begin{aligned}
& J_{11}=1+\frac{k\left[a(1-b)+c_{1}-4 p_{1}+b p_{2}\right]}{1-b^{2}}, \\
& J_{12}=\frac{b k p_{1}}{1-b^{2}}, \\
& J_{21}=\frac{k b p_{2}\left(1-b^{2}\right)+b k^{2} p_{2}\left[a(1-b)+c_{1}-b c_{2}+2 b p_{2}-4 b p_{1}\right]}{\left[1-b^{2}\right]^{2}}, \\
& J_{22}=1+\frac{k\left[a(1-b)+c_{2}+b p_{1}-4 p_{2}\right]}{1-b^{2}}+\frac{b p_{1}\left[a(1-b)+c_{2}+b p_{1}-4 p_{2}\right] k^{2}}{\left[1-b^{2}\right]^{2}} .
\end{aligned}
$$

The stability of the above fixed points depends on the eigenvalues $\lambda_{1}$ and $\lambda_{2}$ of the Jacobian (10). These eigenvalues have the following properties:

(1) A fixed point is a locally stable attracting node if its eigenvalues satisfy $\left|\lambda_{1,2}\right|<1$

(2) A fixed point is an unstable repelling node if its eigenvalues satisfy $\left|\lambda_{1,2}\right|>1$

(3) A fixed point is a saddle point if its eigenvalues satisfy $\left|\lambda_{1}\right|<1$ and $\left|\lambda_{2}\right|>1$ (or $\left|\lambda_{1}\right|>1$ and $\left|\lambda_{2}\right|<1$ )

(4) A fixed point is a nonhyperbolic point if its eigenvalues satisfy $\left|\lambda_{1}\right|=1$ and $\left|\lambda_{2}\right| \neq 1$ (or $\left|\lambda_{1}\right| \neq 1$ and $\left.\left|\lambda_{2}\right|=1\right)$

Proposition 1. The fixed point $E_{0}$ is an unstable repelling point. 
Proof. The Jacobian at this point becomes

$$
J_{E_{0}}=\left(\begin{array}{cc}
1+\frac{k\left[a(1-b)+c_{1}\right]}{1-b^{2}}, & 0, \\
0, & 1+\frac{k\left[a(1-b)+c_{2}\right]}{1-b^{2}},
\end{array}\right)
$$

whose eigenvalues are $\lambda_{i}=1+\left(\left(k\left[a(1-b)+c_{i}\right]\right) /\left(1-b^{2}\right)\right), i=1,2$. Since $|b|<1$ and $c_{i}>0$, we have $\left|\lambda_{i}\right|>1$. This completes the proof.

Proposition 2. The point $E_{1}$ is a stable point provided that $0<\left(\left(k\left[a(1-b)+c_{2}\right]\right) /\left(1-b^{2}\right)\right)<2$ $0<\left(\left(k\left[a(1-b)(2+b)+2 c_{1}+b c_{2}\right]\right) /\left(1-b^{2}\right)\right)<4$.

Proof. The Jacobian at $E_{1}$

$$
J_{E_{1}}=\left(\begin{array}{cc}
1+\frac{k\left[a(1-b)(2+b)+2 c_{1}+b c_{2}\right]}{2\left[1-b^{2}\right]}, & 0, \\
\frac{b k\left[a(1-b)+c_{2}\right]\left[\left(1-b^{2}\right)+k\left(a+c_{1}\right)-a b^{2} k\right]}{2\left[1-b^{2}\right]^{2}}, 1-\frac{k\left[a(1-b)+c_{2}\right]}{1-b^{2}},
\end{array}\right)
$$

has two real eigenvalues

$$
\begin{aligned}
& \lambda_{1}=1-\frac{k\left[a(1-b)+c_{2}\right]}{1-b^{2}}, \\
& \lambda_{2}=1-\frac{k\left[a(1-b)(2+b)+2 c_{1}+b c_{2}\right]}{2\left(1-b^{2}\right)} .
\end{aligned}
$$

The conditions $\left|\lambda_{1}\right|<1$ and $\left|\lambda_{2}\right|<1$ complete the proof. The same proof is for $E_{2}$.

Proposition 3. At $a=2, b=0.2, c_{1}=1, c_{2}=0.3$, and $k=0.5$, we have $E_{3}=(1.41028,1.10282)$. It is locally asymptotically stable.

Proof. The Jacobian at those parameters and $E_{3}$ is

$$
J_{E_{3}}=\left(\begin{array}{cc}
-0.46904 & 0.14690 \\
-0.044276 & -0.11502
\end{array}\right),
$$

and the eigenvalues are $\lambda_{1} \approx-0.4496$ and $\lambda_{2} \approx-0.13446$ with $\left|\lambda_{1,2}\right|<1$, and then $E_{3}$ is stable. It is easy to see that the determinant of $J_{E_{3}}$ equals $\delta=0.060454<1$ which means that map (6) is dissipative. This numerical proof of the stability of $E_{3}$ gives rise to more studies in order to investigate and get more insights on the regions on where this fixed point can be destabilized. This will be discussed in the simulation given in the next section.

3.2. Dynamic Analysis via Numerical Simulation. Here, we investigate the influences of parameter $k$ on the dynamics of map (6). This makes us to assume different sets of parameter values as follows.

3.2.1. Set 1. In this set, we assume the following values: $a=2, b=0.2, c_{1}=1, c_{2}=0.3$, and $k=0.5$. This set indicates that we have two firms with different marginal costs $\left(c_{1}>c_{2}\right)$. In this set, we have $E_{3}=(1.41028,1.10282)$. This requires to investigate the impact of one parameter of those parameters on the stability of this point. Let us assume that parameter $k$ will be taken as the bifurcation parameter. The first numerical experiment displayed in Figure 1(a) shows the effect of bifurcation parameter $k$ on the stability of $E_{3}$. One can see that it is locally stable for all the values of $k$ except for $k \approx 0.704$ on which the first period- 2 cycle is born. Periodic cycles may possess complicated basins of attraction, and this makes us to analyze these basins for cycles. At $k=0.88$, a period 2 with its basins is given in Figure $1(\mathrm{~b})$ with the fixed point $E_{3}$. From now on, we will fix the colors as follows. The grey color denotes the divergent and nonconvergent points, while the other two colors refer to the basins of the plotted cycle. Increasing $k$ further to 0.915 of a period-4 cycle rises with quite complicated basins of attraction than those of period-2 cycle. Figure 1(d) shows complicated basins for period- 8 cycle at $k=0.924$. Further increase in the parameter gives rise to more complicated attractive basins of higher periodic cycles, for instance, at $k=0.95$, the dynamic changes into two disconnected chaotic areas which gather together to become one chaotic attractor at $k=0.995$. Figures 1 (e) and 1(f) present those chaotic behaviors of map (6).

Using the parameter values in set 1 , it is observed through numerical experiments that when the marginal costs are equal $\left(c_{1}=c_{2}\right)$, another dynamic behavior is emerged. At $c_{1}=c_{2}=1$, the fixed point $E_{3}$ can be destabilized via Neimark-Sacker bifurcation. The Jacobian (10) at these parameter values becomes

$$
J_{E_{3}}=\left(\begin{array}{cc}
-0.80659 & 0.18066 \\
-0.13619 & -0.75027
\end{array}\right),
$$

with complex eigenvalues $\lambda_{1,2}=-0.77843 \pm 0.15431 i$ and $\left|\lambda_{1,2}\right|=0.79358<1$, and then $E_{3}$ is locally stable. It is easy to see that the determinant of $J_{E_{3}}$ equals $\delta=0.62976<1$ which means that map (6) is also dissipative. Now, we analyze the influence of $k$ on the stability of this point. When we increase $k$ further, dynamic behaviors arise such as spiral and 

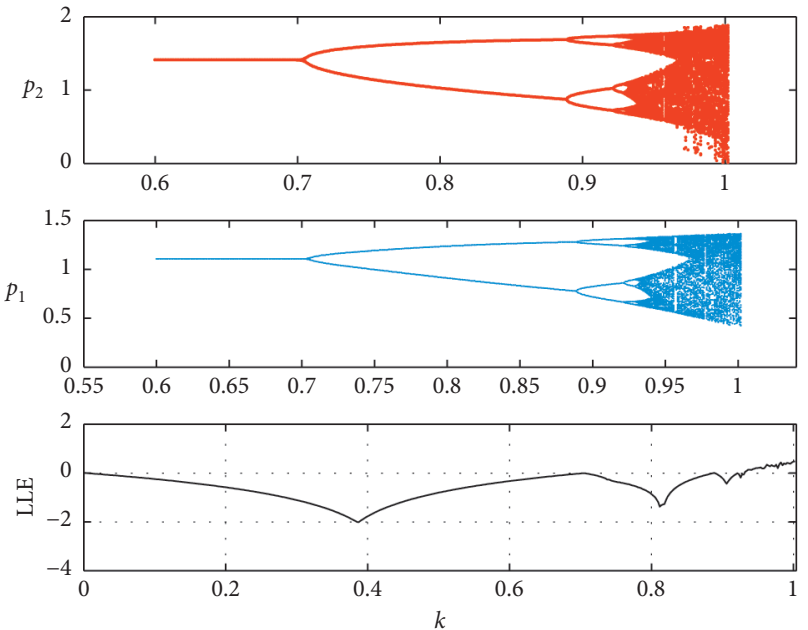

(a)

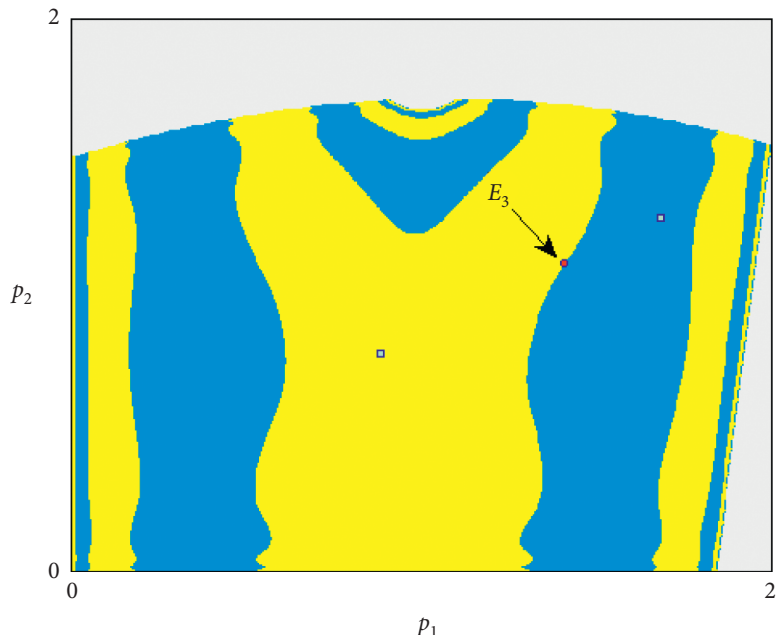

(b)
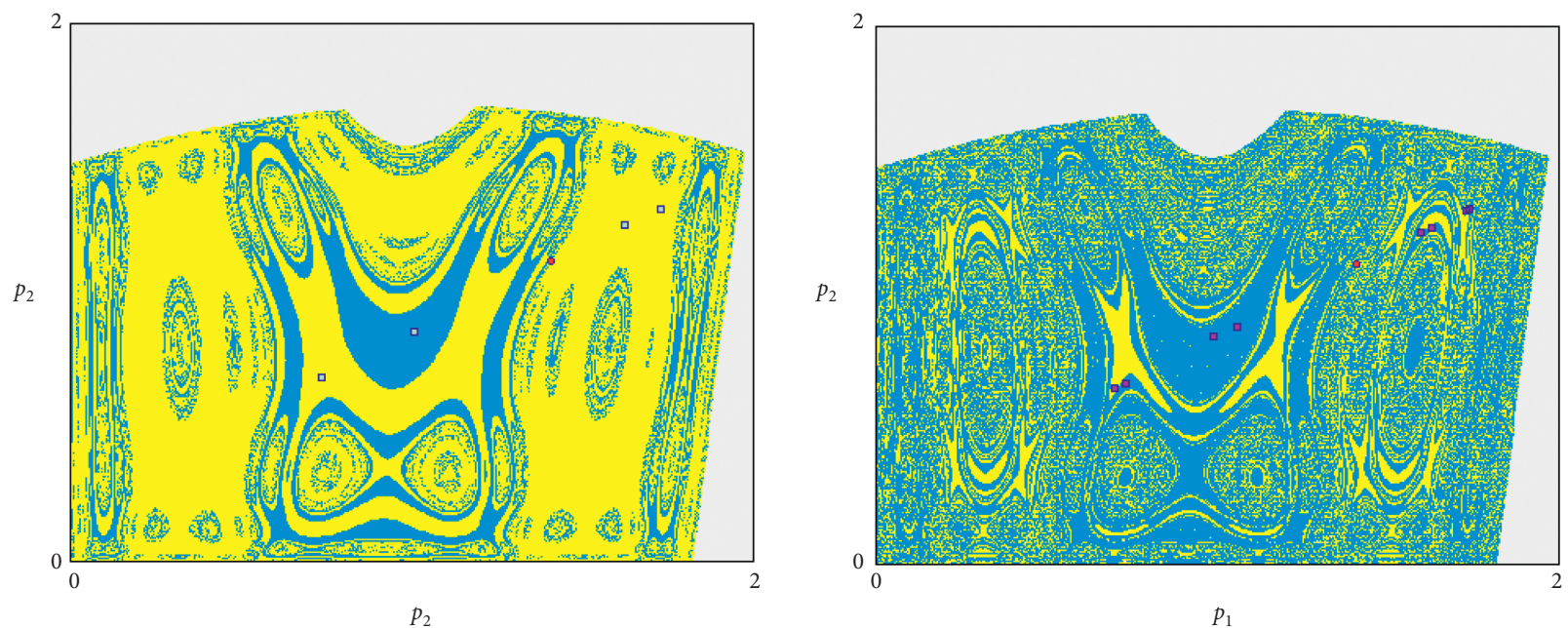

(c)

(d)

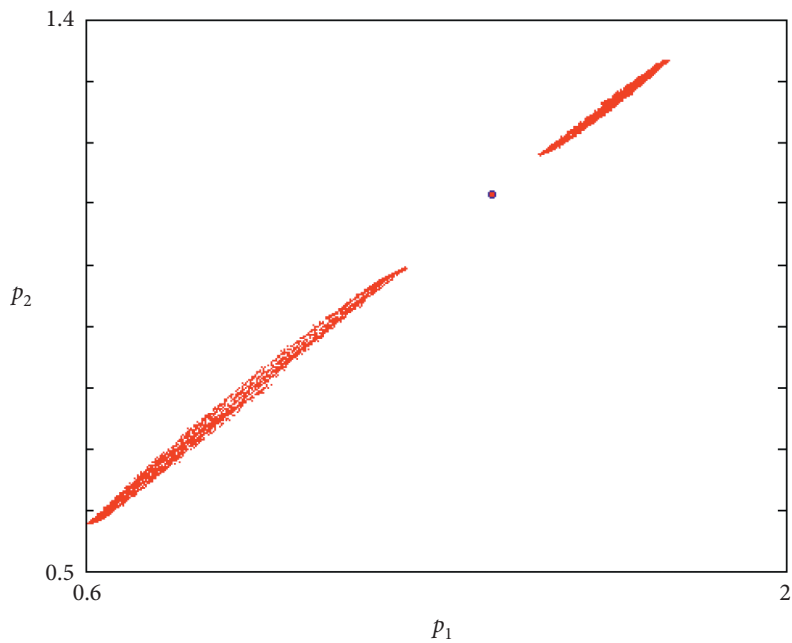

(e)

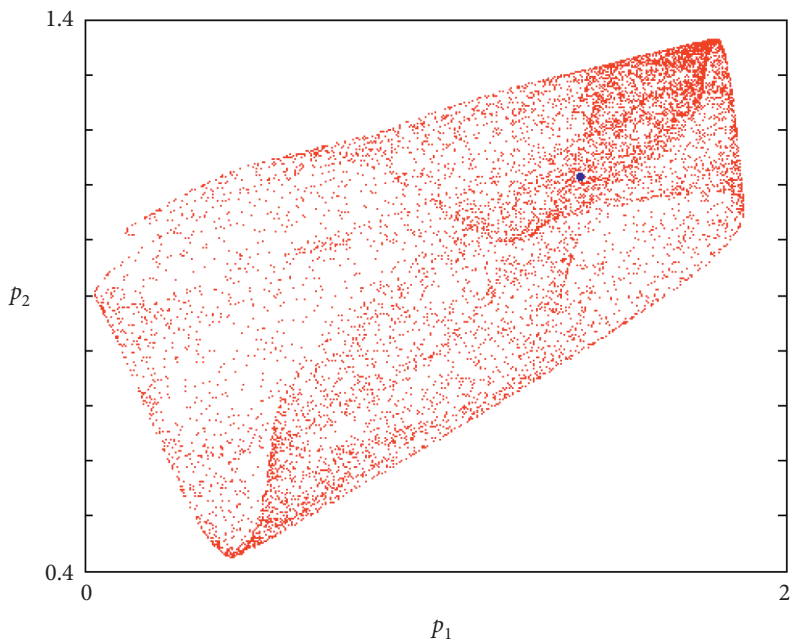

(f)

Figure 1: (a) Flip bifurcation diagram on varying $k$. (b) Basins of attraction of the period-2 cycle at $k=0.88$. (c) Basins of attraction of the period- 4 cycle at $k=0.915$. (d) Basins of attraction of the period- 8 cycle at $k=0.924$. (e) The phase plane of the two chaotic attractors at $k=0.95$. (f) The phase plane of the chaotic attractor at $k=0.995$. Other parameter values are $a=2, b=0.2, c_{1}=1$, and $c_{2}=0.3$. 
closed curves around the fixed point. Figure 2(b) gives those behaviors for different values of $k$. When $k$ reaches 0.774 , a period-17 cycle appears with quite complicated basins of attraction as given in Figure 2(c). As $k$ increases further, high-periodic cycles coexist with complicated basins until the dynamic turns into a closed chaotic attractor. This chaotic attractor is born at $k=0.79$, and its phase plane is displayed in Figure 2(d). This chaotic attractor becomes very complicated as $k$ increases to 0.818 , and its phase plane is shown in Figure 3(a). This unexpected dynamic gives no predictions about the evolution of map (6) and makes the price game with asymmetric information more complicated. The asymmetry and nonlinearity of this map give more complicated behaviors that we should report here. At $k=0.827$, a six-piece chaotic attractor is emerged. The phase plane of this dynamic behavior around the fixed point is given in Figure 3(b). In this figure, we enlarge one piece of this chaotic behavior. These six pieces are gathered together as $k$ increases further to form one chaotic and complicated attractor in Figure 3(c). At $k=0.877$, a period-7 cycle coexists. As given in Figure 3(d), it has very complicated attractive basins. Increasing $k$ to 0.88 , another period cycle is raised. This period is of cycle 14, and its basins are plotted in Figure 3(e). Finally, for any other increase for $k$, the dynamic behavior of map (6) turns to one chaotic attractor. Figure 3(f) displays the phase plane of this chaotic attractor.

3.2.2. Set 2. In this set, we assume the following values: $a=$ $b=c_{1}=c_{2}=0.5$ and $k=1.6$. The fixed point at set 2 becomes $E_{3}=(0.5,0.5)$, and its Jacobian is

$$
J_{E_{3}}=\left(\begin{array}{cc}
-1.1333 & 0.53333 \\
-0.60444 & -0.56444
\end{array}\right)
$$

with complex eigenvalues $\lambda_{1,2}=-0.84889 \pm 0.49139 i$ and $\left|\lambda_{1,2}\right|=0.98086<1$, and then $E_{3}$ is locally stable. It is easy to see that the determinant of $J_{E_{3}}$ equals $\delta=0.96207<1$ which means that map (6) is again dissipative. As previously stated, we study the effects of $k$ on the stability of the fixed point. It is not a repeated analysis for the above simulation; it gives other interesting and hidden dynamics of map (6). Increasing $k$ makes the fixed point unstable due to Neimark-Sacker bifurcation. For instance, at different values of $k$, we give in Figure 4(a) different closed curves around the fixed point. Those closed invariant curves are changed to a period- 5 cycle as parameter $k$ increases to 1.83 . It is plotted in Figure 4(b) with its attractive basins. Another interesting observation is the coexistence of period- 5 and period- 10 cycles together at $k=1.9$. They have quite complicated basins of attraction which are given in Figure 4(c). Increasing $k$ further makes those cycles convert into five unconnected and complicated chaotic areas. The phase plane of these chaotic areas is given in Figure 4(d) with the fixed point. These chaotic areas become larger and more complicated as $k$ increases to 1.95 . They are displayed in
Figure 4(e). After that, the dynamic behavior of map (6) becomes chaotic as $k$ increases to 1.97 , as given in Figure 4(f).

We should highlight here that the role of parameter $k$ is affected by parameter $b$. Figure 5(a) shows that the fixed point can be destabilized due to flip bifurcation on varying parameter $k$ and for the same set of parameter values except for $b=-0.55$. We give some numerical simulations of the dynamic behavior of $k$ at negative values for $b$. At $k=1.51$, Figure 5(b) shows the coexistence of unconnected period-3 cycles. It has an attractive basins given in Figure 5(b) which are more complicated than those obtained previously. At $b=-0.5$, the effect of $k$ on the fixed point makes it unstable due to flip bifurcation. Figure 5(c) shows a stable fixed point for all $k$ until the coexistence of the unconnected period- 6 cycle. This cycle has basins of attraction which are given in Figure 5(d).

3.3. Map (6) versus Map (7). Here, we compare the two models in order to investigate the influences of asymmetric information in their dynamics. Assuming that $a=2, b=0.2, c_{1}=1$, and $c_{2}=0.3$, Figure 6(a) shows the impact of $k$ on map (7). Comparing Figure 1(a) with Figure 6(a), we see that the asymmetric information possessed by the second firm broadens the stability region for both players simultaneously. Numerical experiments show that the basins of attraction detected for map (6) are quite complicated than those of map (7). Furthermore, we show by experiments that, at sets 1 and 2 , the stability region of the fixed point for map (5) is bigger than that detected for the fixed point of map (7). For more details about the properties of classic model (7), refer [31]. In Figure 6(b), we present the attractive basins of the period-4 cycle for map (7). Comparing these basins with others given in Figure 1(c), we get that the dynamics of map (6) are more complex than those of map (7). Therefore, the future prediction for map (6) becomes more complicated.

The previous analysis shows that the structure of basins is quite complicated, and this requires to study extra characteristics of these maps. Setting $p_{1, t+1}=p_{1}$ and $p_{2, t+1}=$ $p_{2}$ in (6), where 1 refers to the time evolution, we get

$$
T\left(p_{1}, p_{2}\right):\left\{\begin{aligned}
p_{1}= & p_{1}+\frac{k p_{1}}{1-b^{2}}\left[a(1-b)+c_{1}-2 p_{1}+b p_{2}\right], \\
p_{2}= & p_{2}+\frac{k p_{2}}{1-b^{2}}\left[a(1-b)+c_{2}+b p_{1}-2 p_{2}\right] \\
& +\frac{k^{2} b p_{1} p_{2}}{\left(1-b^{2}\right)^{2}}\left[a(1-b)+c_{1}-b c_{2}-2 p_{1}+2 b p_{2}\right] .
\end{aligned}\right.
$$

Complicated nonlinear map (18) makes us to study its noninvertibility characteristic by substituting $a=2, b=0.2, c_{1}=1, c_{2}=0.3$, and $k=0.88$ in (18) as follows: 

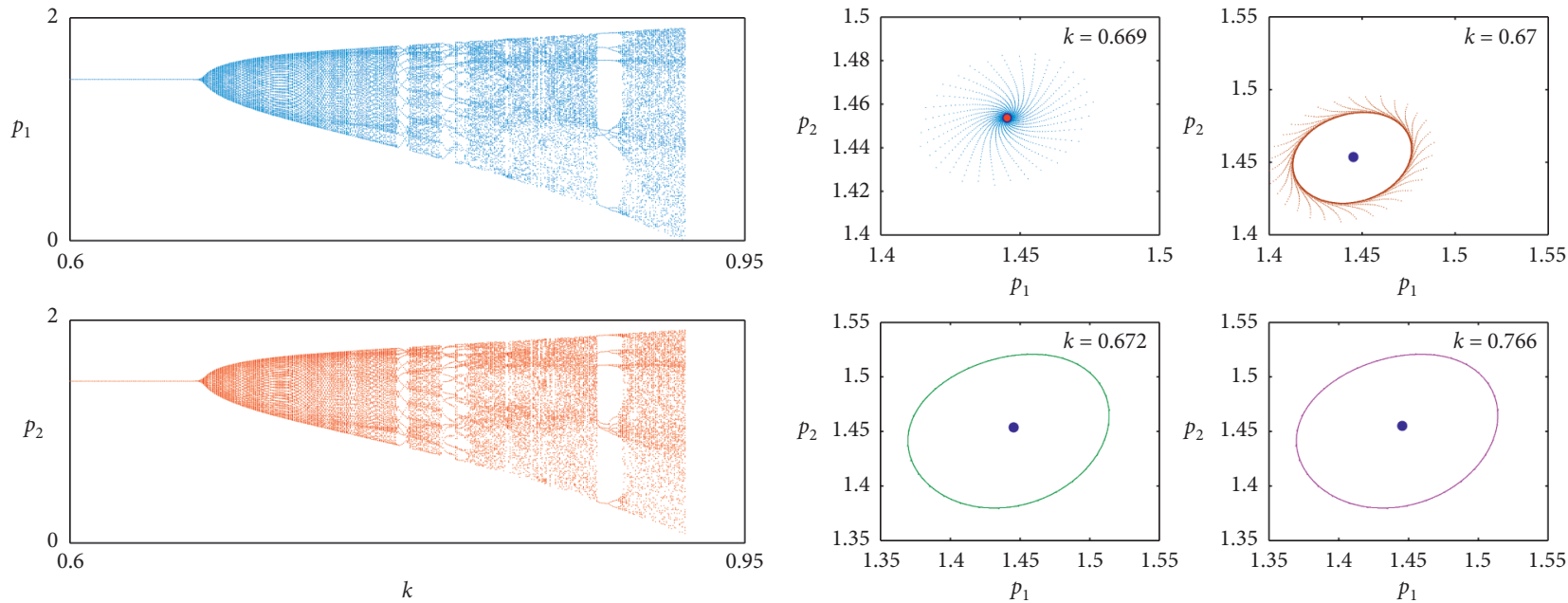

(a)

(b)
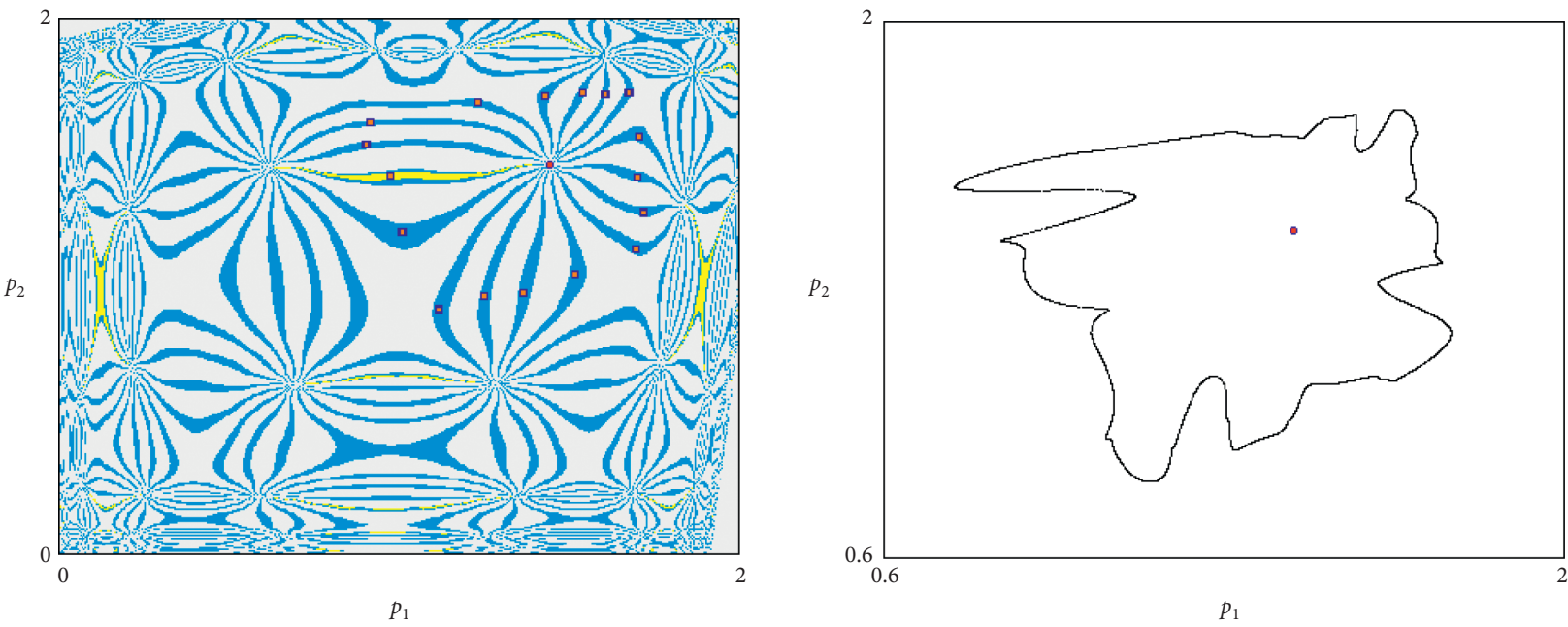

(c)

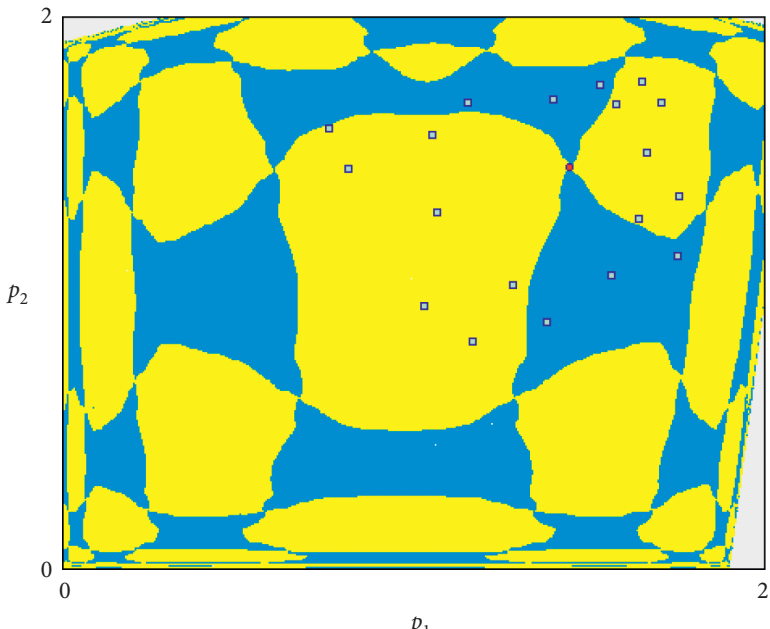

(e)

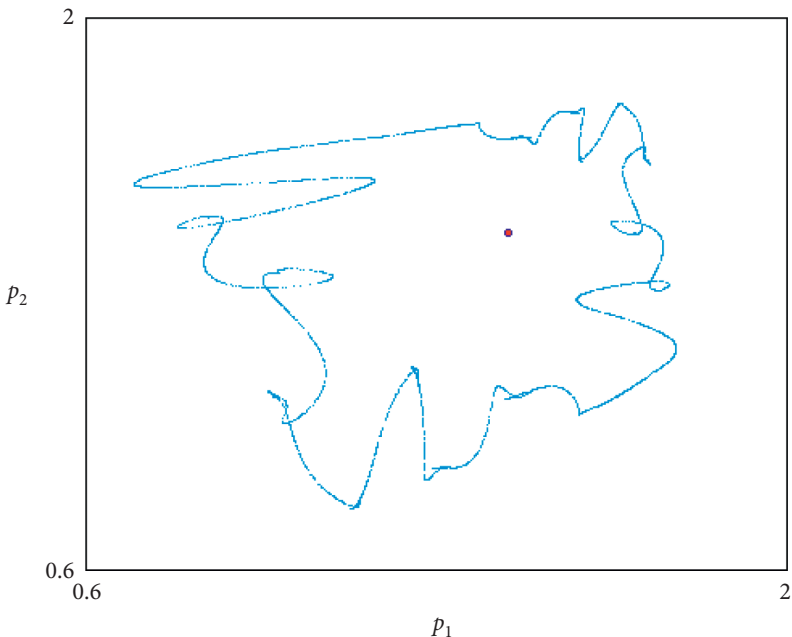

(f)

Figure 2: (a) Neimark-Sacker bifurcation diagram on varying $k$. (b) The phase plane of spiral and closed curves around the fixed point for different values of $k$. (c) Basins of attraction of the period-17 cycle at $k=0.774$. (d) The phase plane of the chaotic attractor at $k=0.79$. (e) Basins of attraction of the period- 19 cycle at $k=0.795$. (f) The phase plane of the chaotic attractor at $k=0.8$. Other parameter values are $a=2, b=0.2, c_{1}=1$, and $c_{2}=1$. 


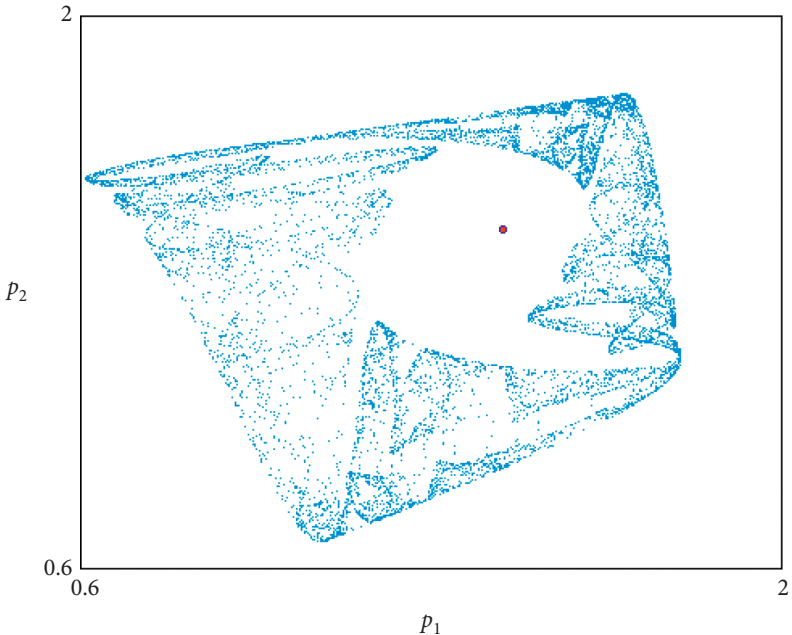

(a)

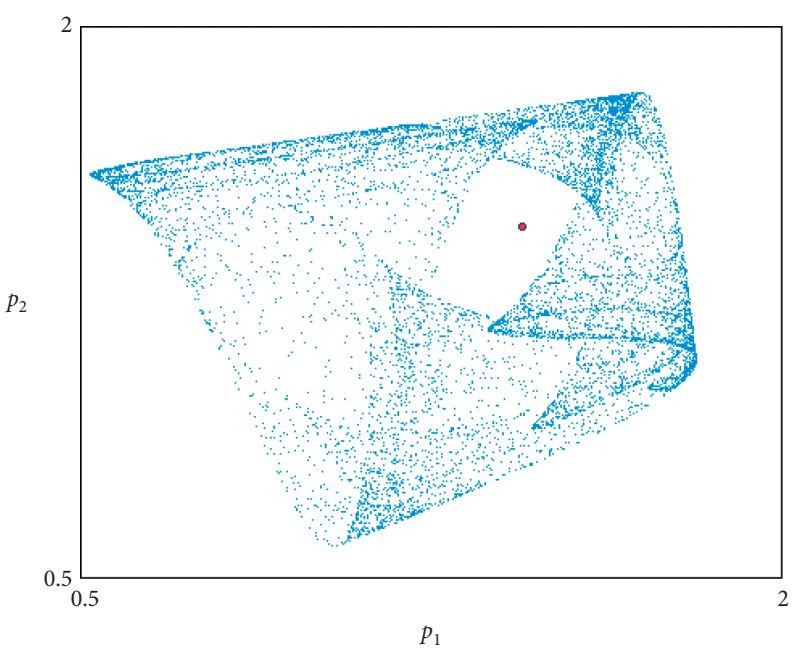

(c)

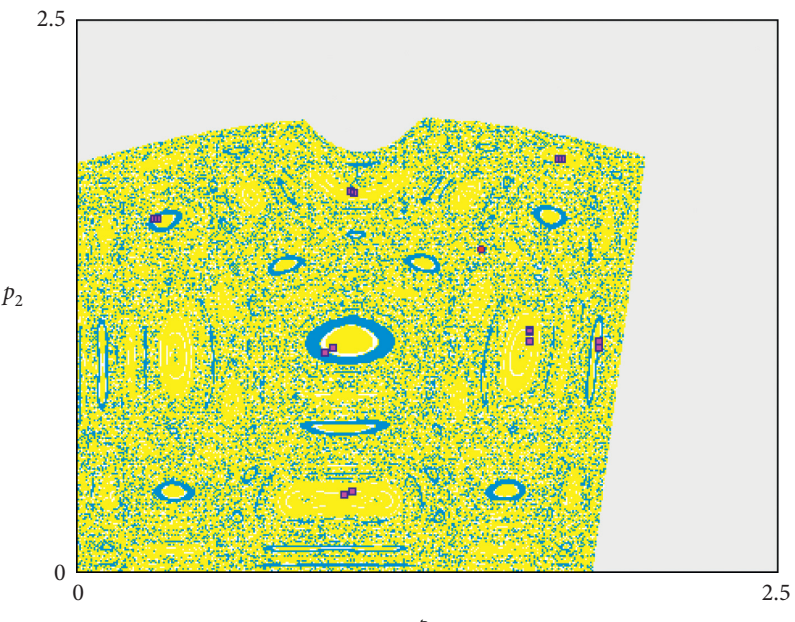

(e)

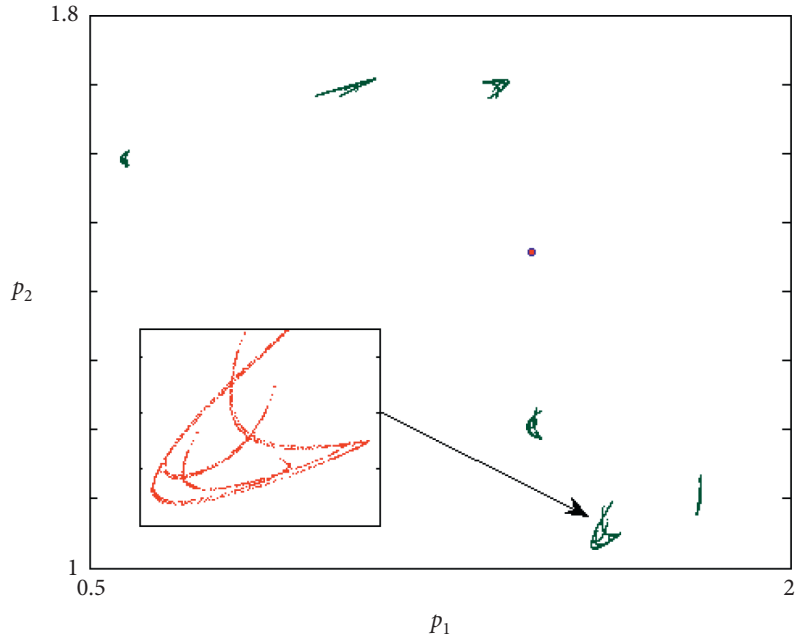

(b)

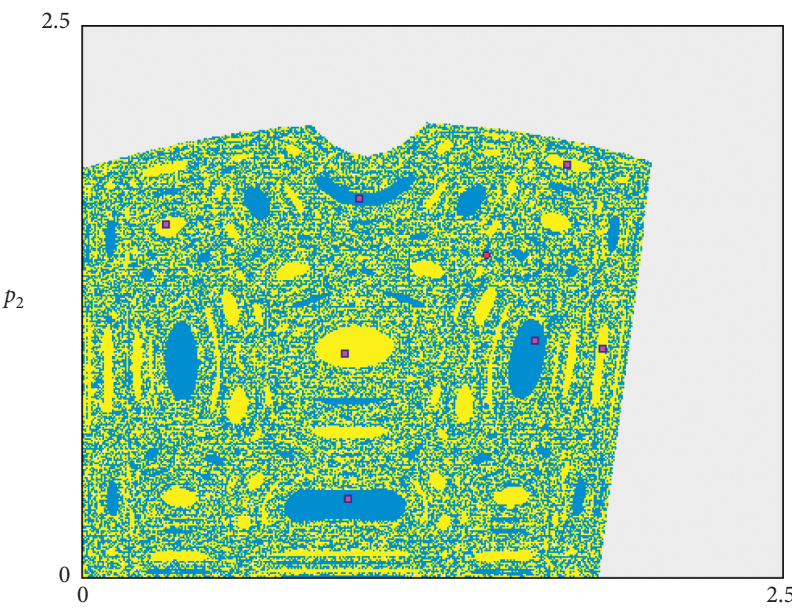

$p_{1}$

(d)

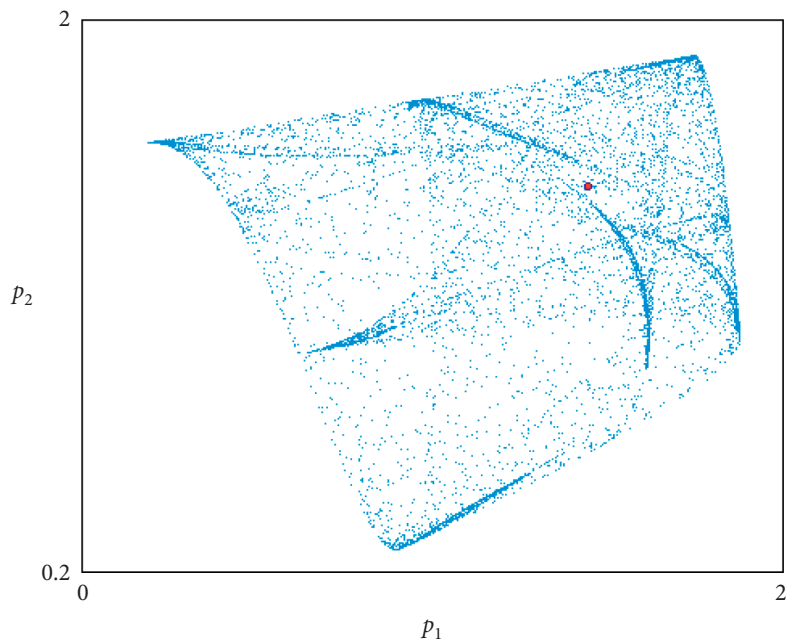

(f)

Figure 3: (a) The phase plane of the chaotic attractor at $k=0.818$. (b) The phase plane of six-piece chaotic attractors at $k=0.827$. (c) The phase plane of the chaotic attractor at $k=0.835$. (d) Basins of attraction of the period-7 cycle at $k=0.877$. (e) Basins of attraction of the period-14 cycle at $k=0.88$. (f) The phase plane of the chaotic attractor at $k=0.89$. Other parameter values are $a=2, b=0.2, c_{1}=1$, and $c_{2}=1$. 

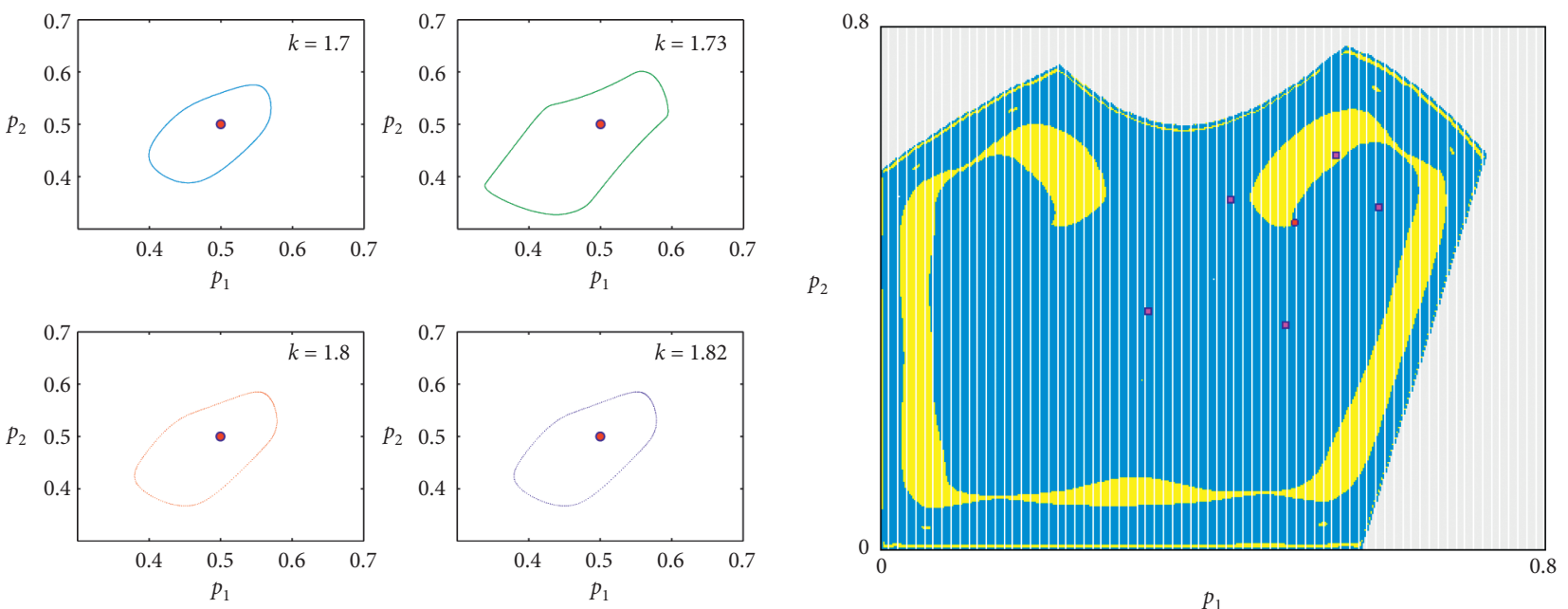

(a)

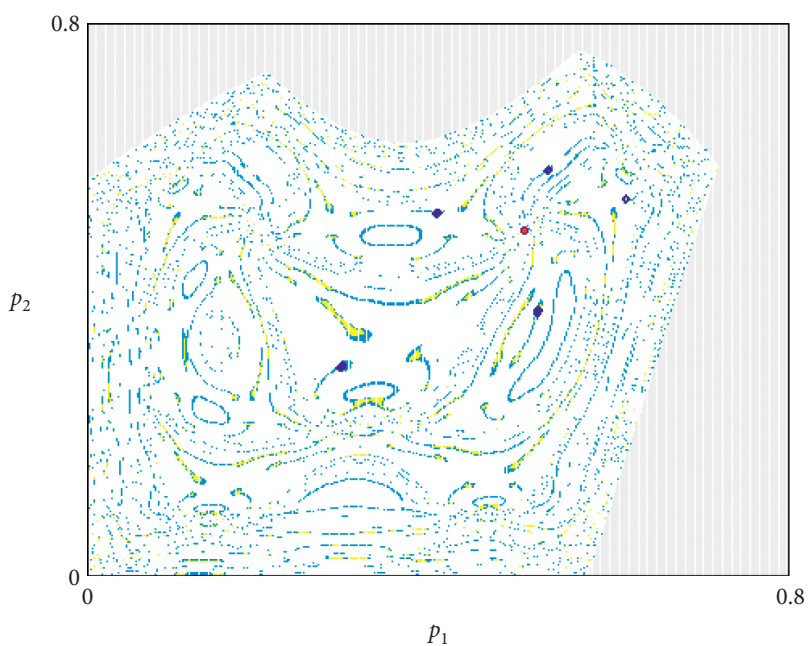

(c)

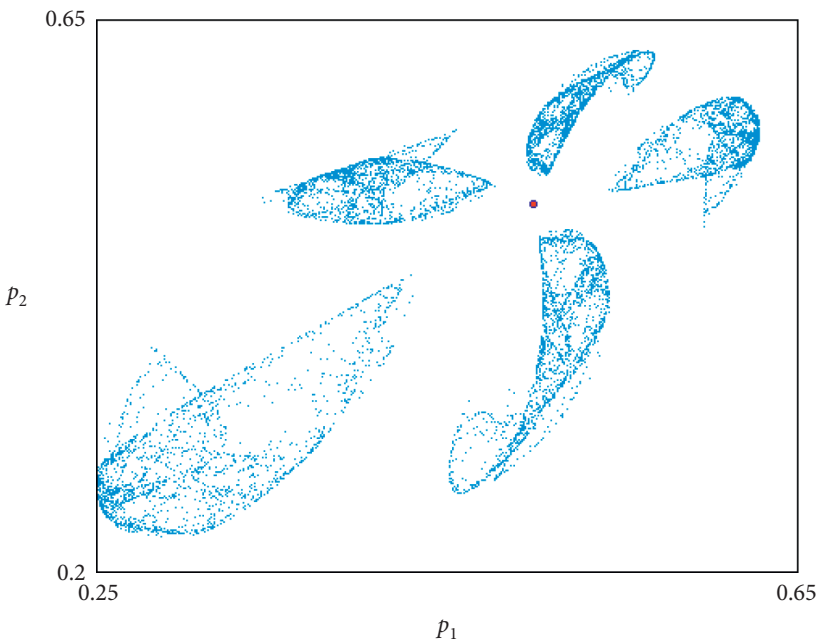

(e)

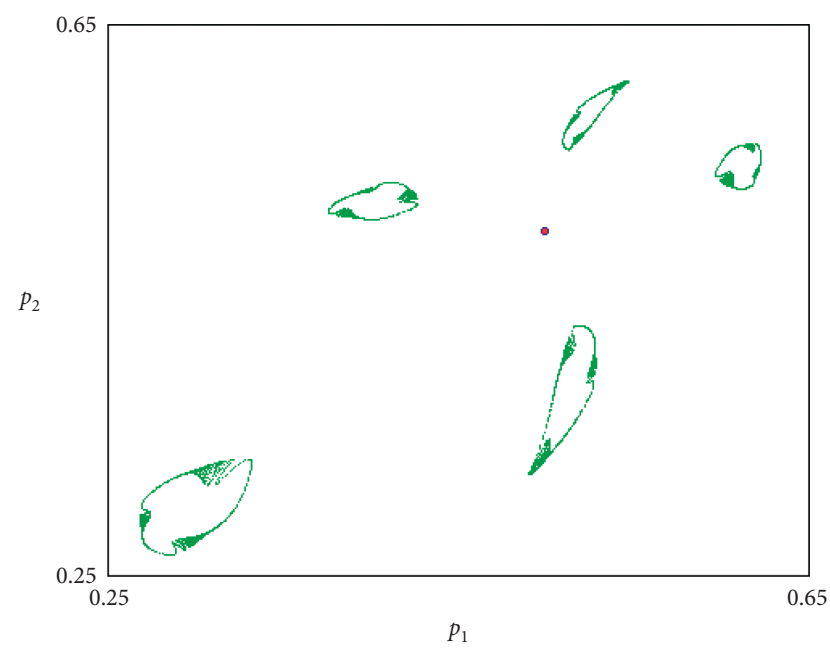

(d)

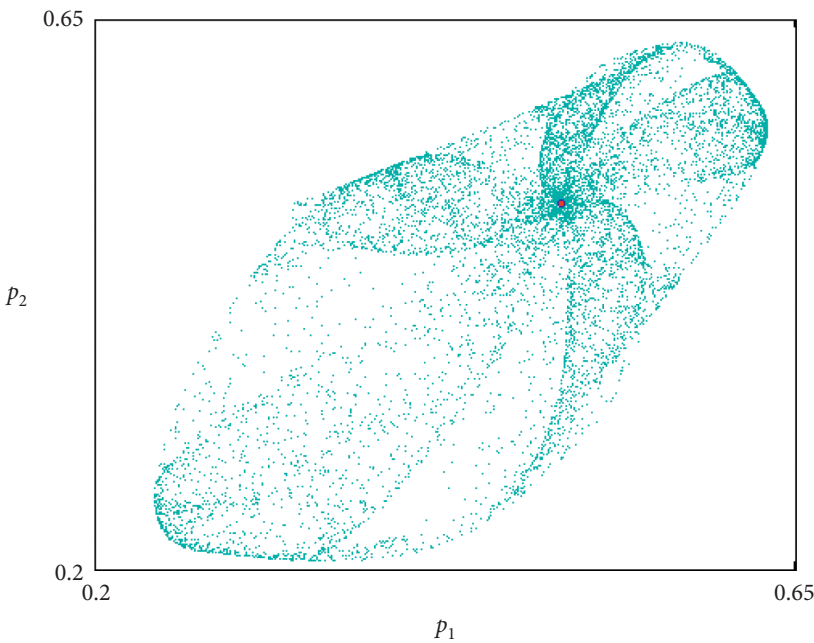

(f)

Figure 4: (a) The phase plane of closed invariant curves at different values of $k=0.818$. (b) Basins of attraction of the period-5 cycle at $k=1.83$. (c) Basins of attraction of period-5 and period-10 cycles at $k=1.9$. (d) The phase plane of five-piece chaotic attractors at $k=1.92$. (e) The phase plane of five-piece chaotic attractors at $k=1.95$. (f) The phase plane of the chaotic attractor at $k=1.97$. Other parameter values are $a=0.5, b=0.5, c_{1}=0.5$, and $c_{2}=0.5$. 


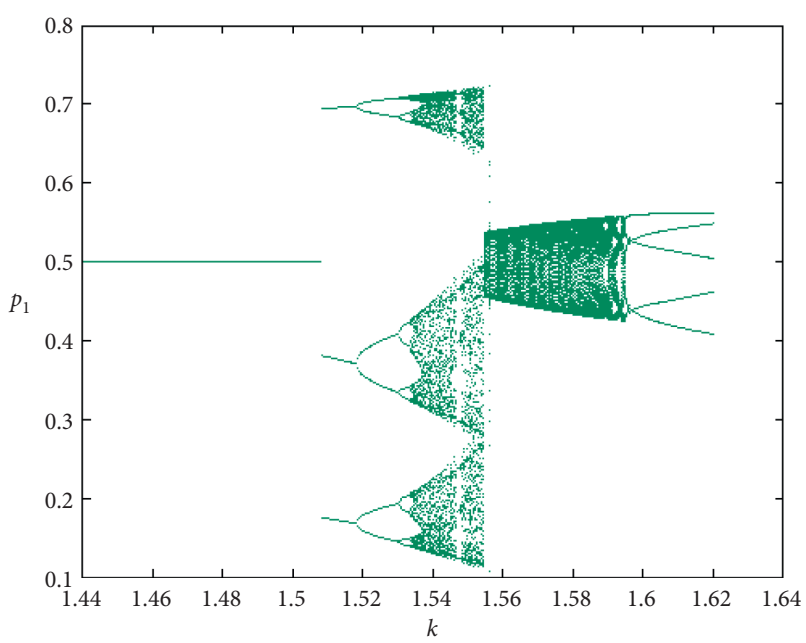

(a)

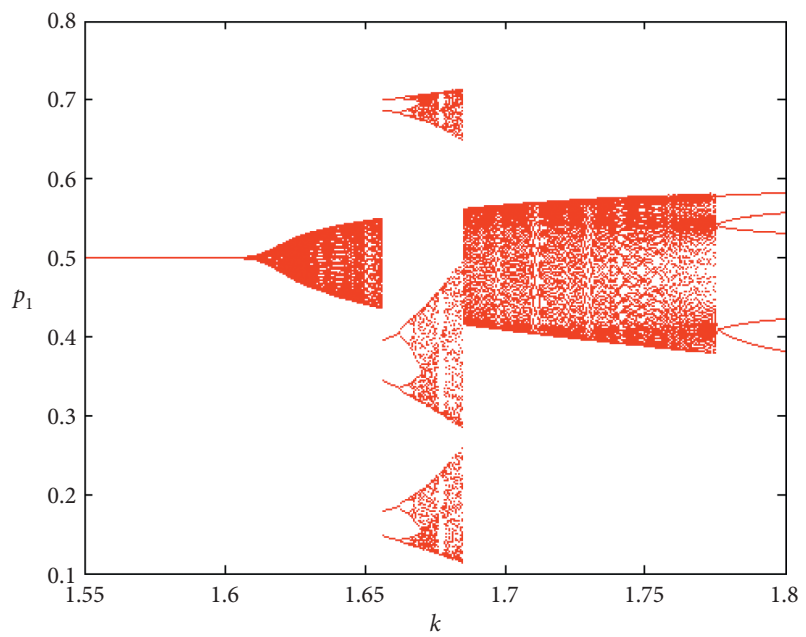

(c)

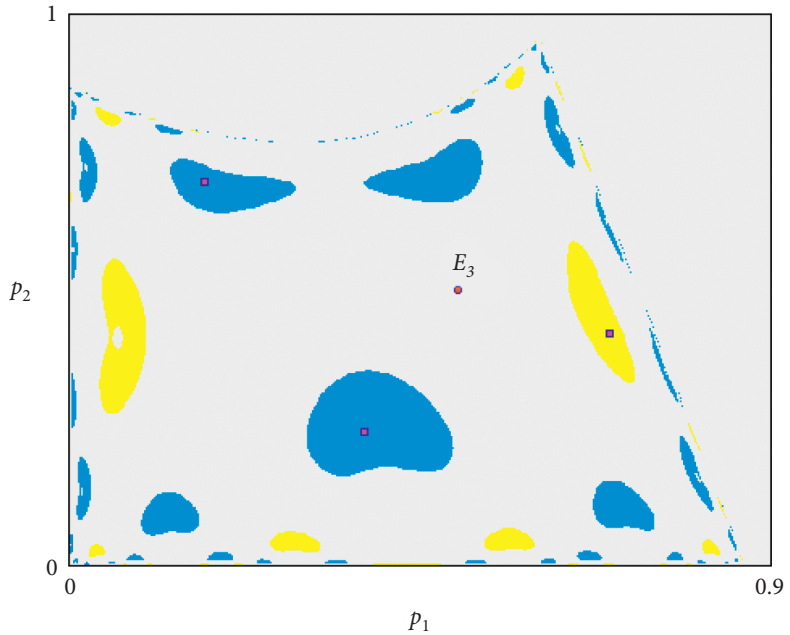

(b)

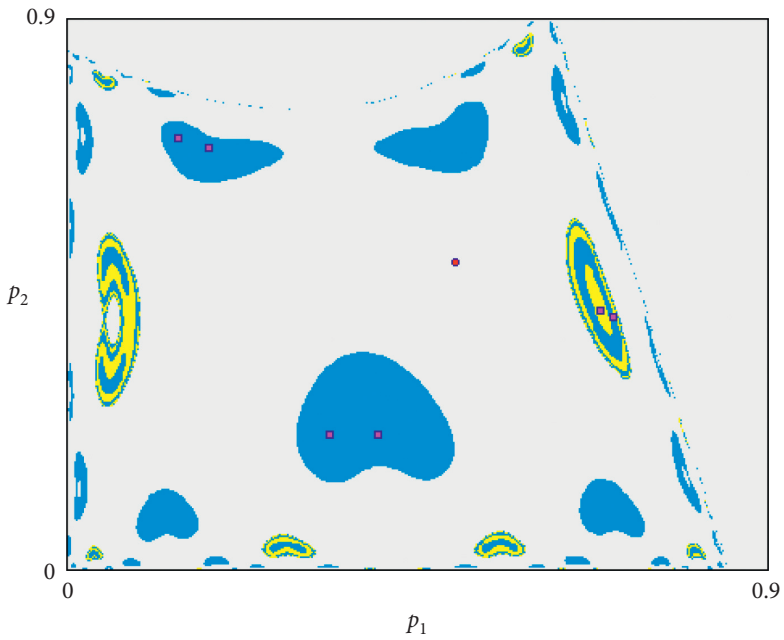

(d)

Figure 5: (a) The flip bifurcation on varying $k$ at $b=-0.55$. (b) The basins of attraction of the period-3 cycle at $k=1.51$ and $b=-0.55$. (c) The flip bifurcation on varying $k$ at $b=-0.5$. (d) The basins of attraction of the period- 6 cycle at $k=1.66$ and $b=-0.5$. The other parameter values are $a=0.5, c_{1}=0.5$, and $c_{2}=0.5$.
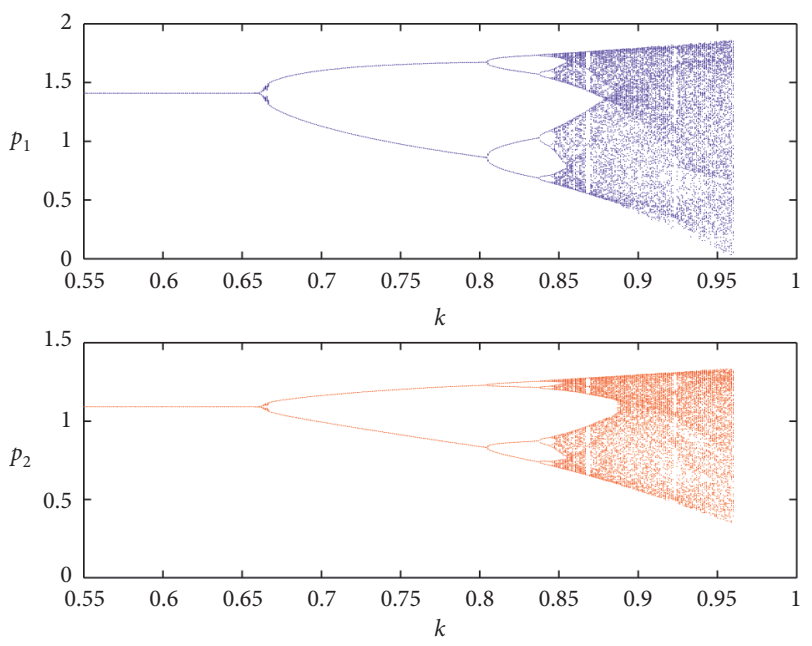

(a)

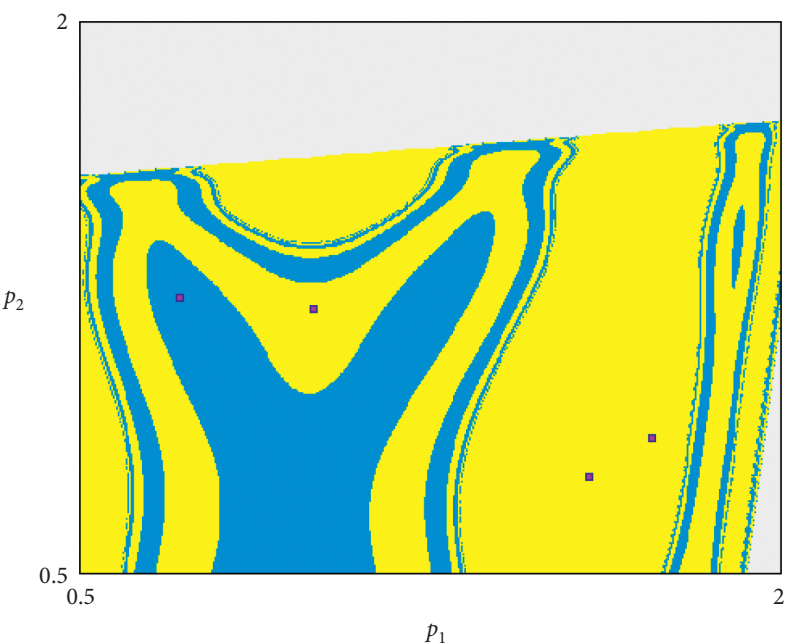

(b)

Figure 6: (a) Bifurcation diagram for map (7) on varying $k$. (b) The basins of attraction for the period-4 cycle of map (7) at $k=0.70$. Other parameters are $a=2, b=0.2, c_{1}=1$, and $c_{2}=0.3$. 


$$
T\left(p_{1}, p_{2}\right):\left\{\begin{aligned}
\dot{p_{1}}= & p_{1}\left(2.2833-1.8333 p_{1}+0.18333 p_{2}\right), \\
\hat{p}_{2}= & p_{2}\left(1.6417+0.40853 p_{1}+0.067222 p_{1} p_{2}\right. \\
& \left.-1.8333 p_{2}-0.33611 p_{1}^{2}\right) .
\end{aligned}\right.
$$

This means that a point $\left(p_{1}, p_{2}\right)$ can be characterized by many rank-1 preimages. Those preimages are mapped in $\left(p_{1}, p_{2}\right)$ in one iteration. Still, map (19) is in a complicated form to solve it algebraically with respect to the variables $p_{1}$ and $p_{2}$. Here, we follow the same procedure carried out in [28] by calculating the number of preimages of points belonging to the, vertical axis $\left(p_{1}=0\right)$. Therefore, substituting the point $\left(0, p_{2}\right)$ in $(13)$, we get

$$
p_{1}=0 \text { or } p_{1}=1.245459+0.1 p_{2} \text {. }
$$

This means if we substitute $p_{1}=0$ in the second part of (13), we get two real preimages for the point $\left(0, p_{2}\right)$ provided that $p_{2} \in(-\infty, 0.36753)$; otherwise, we have no preimages. This concludes that $T$ is a noninvertible map of type $Z_{0}-Z_{2}$, and hence, the phase plane is divided into two regions that are $Z_{0}$ and $Z_{2}$. On the contrary, substituting $p_{1}=1.245459+0.1 p_{2}$ in the second part gives that the point $\left(0, p_{2}\right)$ has three real preimages provided that $p_{2} \in(0.37050, \infty)$; otherwise, we have only one real preimage. This means the preimages of the point in this case divide the phase plane into two regions $Z_{1}$ and $Z_{3}$, and hence, the map $T$ is of type $Z_{1}-Z_{3}$. The same procedure can be used for the points belonging to the horizontal axis $\left(p_{2}=0\right)$ and take the form $\left(p_{1}, 0\right)$. It is worth to mention here that both critical lines $L C$ and $L C_{-1}$ are obtained at those parameter values, but they have long forms to be written here or even to be plotted. We have performed the same procedure for map $T_{1}$ for the points $\left(0, p_{2}\right)$ and $\left(p_{1}, 0\right)$. The results show that map $T_{1}$ is of type $Z_{0}-Z_{2}$ only.

\section{Conclusion}

The current paper has introduced and studied a Bertrand duopoly game with asymmetric price information. A gradient-based mechanism that is the bounded rationality has been adopted by game's players to update their prices in the next time step. The interior equilibrium point of the proposed game has been calculated, and its stability conditions have been discussed. We have compared our model with the classical Bertrand model without asymmetric information as both players in the two models use the same gradient-based mechanism. Our contributions have shown that the asymmetric price information has broadened the stability region of the interior point. Furthermore, the structure of basins of attraction for periodic cycles due to this information has shown quite complicated structure for those basins in comparison with those obtained for classical Bertrand. Moreover, the local and global analysis performed have shown that the proposed game's map is noninvertible and belongs to the type of $Z_{0}-Z_{2}$ or $Z_{1}-Z_{3}$ depending on the parameter value sets, while the model of classical Bertrand belongs to the type $Z_{0}-Z_{2}$ only.

\section{Data Availability}

The data used to support the findings of this study are included within the article.

\section{Conflicts of Interest}

The author declares that there are no conflicts of interest.

\section{Acknowledgments}

This work was supported by the Research Supporting Project Number (RSP-2020/167), King Saud University, Riyadh, Saudi Arabia.

\section{References}

[1] https://en.wikipedia.org/wiki/Bertrand_competition.

[2] C. D' Aspremont, J. J. Gabszewicz, and J. F. Thisse, "On Hotelling's stability in competition," Econometrica, vol. 47, no. 5, pp. 1145-1150, 1979.

[3] B. Allen, R. Deneckere, T. Faith, and D. Kovenock, "Capacity precommitment as a barrier to entry: a Bertrand-Edgeworth approach," Economic Theory, vol. 15, no. 3, pp. 501-530, 2000.

[4] W. Elberfeld and E. Wolfstetter, "A dynamic model of Bertrand competition with entry," International Journal of Industrial Organization, vol. 17, no. 4, pp. 513-525, 1999.

[5] J. H. Hamilton, J.-F. Thisse, and A. Weskamp, "Spatial discrimination," Regional Science and Urban Economics, vol. 19, no. 1, pp. 87-102, 1989.

[6] M. Janssen and E. Rasmusen, "Bertrand competition under uncertainty," The Journal of Industrial Economics, vol. 50, no. 1, pp. 11-21, 2003.

[7] A. Ledvina and R. Sircar, "Dynamic Bertrand oligopoly," Applied Mathematics \& Optimization, vol. 63, no. 1, pp. 11-44, 2010.

[8] J. Zhang, Q. Da, and Y. Wang, "The dynamics of Bertrand model with bounded rationality," Chaos, Solitons \& Fractals, vol. 39, no. 5, pp. 2048-2055, 2009.

[9] J. Ma and K. Wu, "Complex system and influence of delayed decision on the stability of a triopoly price game model," Nonlinear Dynamics, vol. 73, no. 3, pp. 1741-1751, 2013.

[10] J. H. Ma and L. Sun, "Study and simulation on discrete dynamics of Bertrand triopoly team-game," Mathematical Problems in Engineering, vol. 2015, Article ID 960380, 12 pages, 2015.

[11] J. Peng, Z. Miao, and f. Peng, "Study on a 3-dimensional game model with delayed bounded rationality," Applied Mathematics and Computation, vol. 218, no. 5, pp. 1568-1576, 2011.

[12] J. Zhao, J. Wei, and Y. Li, "Pricing decisions for substitutable products in a two-echelon supply chain with firms' different channel powers," International Journal of Production Economics, vol. 153, pp. 243-252, 2014.

[13] B. C. Giri and S. Sharma, "Manufacturer's pricing strategy in a two-level supply chain with competing retailers and advertising cost dependent demand," Economic Modelling, vol. 38, pp. 102-111, 2014.

[14] L. Fanti, L. Gori, C. Mammana, and E. Michetti, "The dynamics of a Bertrand duopoly with differentiated products: 
synchronization, intermittency and global dynamics," Chaos, Solitons \& Fractals, vol. 52, no. 1, pp. 73-86, 2013.

[15] S. S. Askar, "On Cournot-Bertrand competition with differentiated products," Annals of Operations Research, vol. 223, no. 1, pp. 81-93, 2014.

[16] S. S. Askar and M. Abouhawwash, "Quantity and price competition in a differentiated triopoly: static and dynamic investigations," Nonlinear Dynamics, vol. 91, no. 3, pp. 1963-1975, 2018.

[17] X. Zhang, D. Sun, S. Ma, and S. Zhang, "The dynamics of a quantum Bertrand duopoly with differentiated products and heterogeneous expectations," Physica A: Statistical Mechanics and Its Applications, vol. 557, Article ID 124878, 2020.

[18] M. Alipranti and E. Petrakis, "Fixed fee discounts and Bertrand competition in vertically related markets," Mathematical Social Sciences, vol. 106, pp. 19-26, 2020.

[19] J. Andaluz, A. A. Elsadany, and G. Jarne, "Nonlinear Cournot and Bertrand-type dynamic triopoly with differentiated products and heterogeneous expectations," Mathematics and Computers in Simulation, vol. 132, pp. 86-99, 2017.

[20] A. Semenov and J.-B. Tondji, "On the dynamic analysis of Cournot-Bertrand Equilibria," Economics Letters, vol. 183, Article ID 10854, 2019.

[21] R. Amir and I. V. Evstigneev, "A new look at the classical Bertrand duopoly," Games and Economic Behavior, vol. 109, pp. 99-103, 2018.

[22] E. Ahmed, A. S. Hegazi, M. F. Elettreby, and S. S. Askar, "On multi-team games," Physica A: Statistical Mechanics and Its Applications, vol. 369, no. 2, pp. 809-816, 2006.

[23] Y. Peng and Q. Lu, "Complex dynamics analysis for a duopoly Stackelberg game model with bounded rationality," Applied Mathematics and Computation, vol. 271, pp. 259-268, 2015.

[24] A. K. Naimzada and R. Raimondo, "Chaotic congestion games," Applied Mathematics and Computation, vol. 321, pp. 333-348, 2018.

[25] J. Ma, L. Sun, S. Hou, and X. Zhan, "Complexity study on the Cournot-Bertrand mixed duopoly game model with market share preference," Chaos, vol. 28, Article ID 023101, 2018.

[26] E. Ahmed and M. F. Elettreby, "Controls of the complex dynamics of a multi-market Cournot model," Economic Modelling, vol. 37, pp. 251-254, 2014.

[27] S. S. Askar, "Tripoly Stackelberg game model: one leader versus two followers," Applied Mathematics and Computation, vol. 328, pp. 301-311, 2018.

[28] F. Cavalli, A. Naimzada, and F. Tramontana, "Nonlinear dynamics and global analysis of a heterogeneous Cournot duopoly with a local monopolistic approach versus a gradient rule with endogenous reactivity," Communications in Nonlinear Science and Numerical Simulation, vol. 23, no. 1-3, pp. 245-262, 2015.

[29] S. S. Askar and K. Alnowibet, "Cooperation versus noncooperation: Cournot duopolistic game based on delay and time-dependent parameters," Chaos, Solitons \& Fractals, vol. 91, pp. 580-584, 2016.

[30] N. Singh and X. Vives, "Price and quantity competition in a differentiated duopoly," The RAND Journal of Economics, vol. 15, no. 4, pp. 546-554, 1984.

[31] S. S. Askar and A. Al-khedhairi, "Dynamic investigations in a duopoly game with price competition based on relative profit and profit maximization," Journal of Computational and Applied Mathematics, vol. 367, Article ID 112464, 2020. 